\title{
Application of Cumulant Method In Performance Evaluation of Turbo-Like Codes
}

\author{
Ali Abedi \\ Electrical and Computer Engineering \\ University of Maine \\ Orono, ME, 04469-5708 \\ Email: abedi@eece.maine.edu
}

\author{
Mary E. Thompson \\ Statistics and Actuarial Science \\ University of Waterloo \\ Waterloo, Ontario, Canada \\ Email: methomps@uwaterloo.ca
}

\author{
Amir K. Khandani \\ Electrical and Computer Engineering \\ University of Waterloo \\ Waterloo, Ontario, Canada \\ E-mail: khandani@uwaterloo.ca
}

\begin{abstract}
In this article, a new method for performance evaluation of Turbo-like codes is presented. This is based on estimating the Probability Density Function $(p d f)$ of the bit Log-Likelihood-Ratio $(L L R)$ using higher order statistics. We do not restrict ourselves to any specific model for the $p d f$ and try to estimate it directly using a Cumulant matching method. Numerical results show a close agreement between the proposed method and simulations. The complexity of this method is similar to the Monte-Carlo simulation with the advantage of providing similar accuracy using significantly fewer samples.
\end{abstract}

\section{INTRODUCTION}

For any arbitrary random variable, the logarithm of its characteristics function may be approximated using a Taylor series expansion. The coefficients of this series expansion are known as Cumulants or higher order statistics. Cumulants have been widely used in a variety of applications including analysis of digital communications systems.

The problem of performance evaluation of coherent optical communication systems is considered in [1], where a solution based on estimating the Cumulants of the noise process is presented. A condition is derived to quantify under what system conditions a Gaussian Probability Density Function $(p d f)$ is a good approximation. A discrete-time method is proposed in [2] for estimating the impulse response of a frequency selective digital modulated communication channel. This method is based on estimating the Cumulants up to the fourth order. Parameters of a moving average model are estimated in [3], using second and third order Cumulant matching. This estimation is further improved in [4]. Cumulants of symmetric distributions like uniform, triangular, and Gaussian are estimated in [5] using a robust estimation technique. The application of Edgeworth series and higher-order statistics to the discretetime detection of a known constant signal in multivariate nonGaussian noise is considered in [6]. A numerical algorithm based on knowledge of the noise Cumulants is presented in order to analyze the finite-sample size performance of the suboptimum detectors.

Non-Gaussian sources are modeled in [7] using Gaussian mixture densities. It is shown that in high Signal to Noise Ratio (SNR) regions, this method outperforms the Cumulant based algorithms for parameter estimation. The problem of blind equalization and estimation of digital communication finite impulse response channels is considered in [8]. The channel parameters are estimated by nonlinear optimization of a quadratic Cumulant matching criterion involving second and fourth order Cumulants. This problem is later considered in [9] for partial-response signals. A method for phase recovery in Quadrature Amplitude Modulation (QAM) communication systems based on higher order statistics is presented in [10]. A relation is derived between the phase error and the fourth order Cumulant of the output.

Since the higher order Cumulant-based criteria can be multimodal, conventional gradient search techniques require a good initial estimate to avoid converging to local minima. This problem is solved in [11], where a novel scheme based on genetic algorithms is employed to optimize the Cumulant fitting cost function. A method based on higher order statistics is proposed in [12] to mitigate the performance degradation caused by multi-path propagation in a mobile radio communication system. It is shown that an over-determined system of linear equations (involving only Cumulants of the received baseband signal) can be obtained to perform non-iterative deconvolution. The study of chaotic communication systems with Additive White Gaussian Noise (AWGN) interference is considered in [13] by employing suitable Cumulant analysis tools.

In this paper we present a method based on using the Cumulants of the bit Log-Likelihood-Ratio $(L L R)$ versus its moments as used in [14]. The first two Cumulants of the normal density are its mean and variance and the higher order Cumulants are zero. Since the $p d f$ of the bit $L L R$ is nearly normal [15]-[17], it is expected that its higher order Cumulants are fairly small. This allows for easy truncation of the series expansion of the $p d f$ in terms of its Cumulants.

This paper is organized as follows. The problem is modeled in Section II. In Section III, the Cumulant matching method, which is used to find the parameters of the proposed model, is described. The accuracy of this method is investigated in Section IV. The numerical results and conclusion are presented in Section V and Section VI, respectively. 


\section{PRELIMINARIES}

A common tool to express the bit probabilities in bit decoding algorithms is based on the so-called $L L R$. The $L L R$ of the $k^{t h}$ bit position is defined by the following equation:

$$
L L R(k)=\log \frac{P\left(c_{k}=1 \mid \mathbf{x}\right)}{P\left(c_{k}=0 \mid \mathbf{x}\right)},
$$

where $c_{k}$ is the value of the $k^{t h}$ bit in the transmitted codeword, $\mathbf{x}$ is the received vector, and $\log$ represents the natural logarithm. Let us define the random variable $Y=L L R(k)$ and let its $p d f$ be denoted as $f(y)$. It is proved in [18] that the $p d f$ of the bit $L L R$ is independent of the transmitted code-word, as long as the value of the bit position under consideration remains unchanged. By using this result and without loss of generality, we consider the case of sending the all-zero code-word. The received bit is decoded to 0 (or 1 ), if the corresponding $L L R$ is negative (or positive). Therefore, the following integral simplifies the remaining Bit Error Rate (BER) calculations:

$$
P_{e}=\int_{0}^{\infty} f(y) d y .
$$

\section{Cumulants Matching}

The characteristic function of a random variable $Y$ with its $p d f$ denoted as $f(y)$ is defined as

$$
\Phi(t)=\int_{-\infty}^{+\infty} f(y) e^{i t y} d y .
$$

The Cumulants of the random variable $Y$, denoted as $k_{m}$, are the coefficients of the following series expansion:

$$
\log \Phi(t)=\sum_{m=0}^{\infty} k_{m} \frac{(i t)^{m}}{m !}
$$

The first few Cumulants can be expressed in terms of the raw moments as follows [19]:

$$
\begin{aligned}
& k_{0}=1, \\
& k_{1}=\mu_{1}, \\
& k_{2}=\mu_{2}-\mu_{1}^{2}, \\
& k_{3}=2 \mu_{1}^{3}-3 \mu_{1} \mu_{2}+\mu_{3},
\end{aligned}
$$

where $\mu_{i}$ is the $i^{\text {th }}$ raw moment of random variable $Y$ with $n$ samples $y_{j}$ defined as

$$
\mu_{i}=\frac{1}{n} \sum_{j=1}^{n} y_{j}^{i}
$$

The $K$-statistics are the unique symmetric unbiased estimators of the Cumulants [20]. Thus,

$$
E\left[K_{m}\right]=k_{m}
$$

where the notation $K_{m}$ is used for the $m^{t h} K$-statistic of a given density. In addition, the variance,

$$
V\left[K_{m}\right]=E\left[\left(K_{m}-k_{m}\right)^{2}\right],
$$

is a minimum compared to all other unbiased estimators [21], [22]. In other words, the $K$-statistics are the Uniformly Minimum Variance Unbiased Estimators (UMVUE) of the Cumulants. The first few $K$-statistics are as follows:

$$
\begin{aligned}
K_{1} & =\frac{S_{1}}{n}, \\
K_{2} & =\frac{n S_{2}-S_{1}^{2}}{n(n-1)}, \\
K_{3} & =\frac{2 S_{1}^{3}-3 n S_{1} S_{2}+n^{2} S_{3}}{n(n-1)(n-2)},
\end{aligned}
$$

where $n$ is the number of samples (denoted by $y_{i}$ ) used in the estimation, and

$$
S_{r}=\sum_{i=1}^{n} y_{i}^{r} .
$$

A combinatorial method for computing higher orders of the $K$ statistics is presented in [23]. Once the first few Cumulants are estimated by using the $K$-statistics, the characteristic function of the bit $L L R$ can be approximated by using (4). Following that, the $p d f$ of the bit $L L R$ can be approximated by taking the Inverse Fourier Transform (IFT) of $\Phi(t)$.

$$
f(y)=\int_{-\infty}^{+\infty} \Phi(t) e^{-i t y} d t
$$

\section{ACCURACY ANALYSIS}

The cumulative distribution function $(C D F)$ of the bit $L L R$ is defined as

$$
F(T)=\int_{-\infty}^{T} f(y) d y .
$$

We are interested in computing the error probability

$$
P_{e}=\int_{0}^{\infty} f(y) d y=1-\int_{-\infty}^{0} f(y) d y=1-F(0) .
$$

Taking the IFT of the characteristic function, $f(y)=$ $\operatorname{IFT}\{\Phi(t)\}$, noting (4), and using properties of IFT for integral of a function, we have

$$
F(T)=\operatorname{IFT}\left\{\frac{1}{i t} \exp \left[\sum_{m=0}^{\infty} k_{m} \frac{(i t)^{m}}{m !}\right]\right\} .
$$

A small error, $\Delta k_{m}$ in estimating each Cumulant, results in an error, $\triangle F(T)$ in $C D F$ :

$$
\begin{aligned}
& F(T)+\Delta F(T)=\operatorname{IFT}\left\{\frac{1}{i t} \exp \left[\sum_{m=0}^{\infty}\left(k_{m}+\Delta k_{m}\right) \frac{(i t)^{m}}{m !}\right]\right\} \\
& =\operatorname{IFT}\left\{\frac{1}{i t} \exp \left[\sum_{m=0}^{\infty} k_{m} \frac{(i t)^{m}}{m !}\right] \exp \left[\sum_{n=0}^{\infty} \Delta k_{n} \frac{(i t)^{n}}{n !}\right]\right\} \quad(21) \\
& \simeq \operatorname{IFT}\left\{\frac{1}{i t} \exp \left[\sum_{m=0}^{\infty} k_{m} \frac{(i t)^{m}}{m !}\right]\left(1+\sum_{n=0}^{\infty} \Delta k_{n} \frac{(i t)^{n}}{n !}\right)\right\} \quad(22) \\
& =F(T)+\operatorname{IFT}\left\{\frac{1}{i t} \exp \left[\sum_{m=1}^{\infty} k_{m} \frac{(i t)^{m}}{m !}\right] \sum_{n=1}^{\infty} \Delta k_{n} \frac{(i t)^{n}}{n !}\right\},
\end{aligned}
$$


taking $\Delta k_{0}=0$. This means,

$$
\begin{aligned}
\Delta F(T) & \simeq \operatorname{IFT}\left\{\frac{1}{i t} \exp \left[\sum_{m=1}^{\infty} k_{m} \frac{(i t)^{m}}{m !}\right] \sum_{n=1}^{\infty} \Delta k_{n} \frac{(i t)^{n}}{n !}\right\} \\
& =\sum_{n=1}^{\infty} \Delta k_{n} \frac{i^{n-1}}{n !} \operatorname{IFT}\left\{t^{n-1} \exp \left[\sum_{m=1}^{\infty} k_{m} \frac{(i t)^{m}}{m !}\right]\right\} \\
& =\sum_{n=1}^{\infty} \Delta k_{n} \frac{i^{n-1}}{n !} f^{(n-1)}(T),
\end{aligned}
$$

where $f^{(n)}(T)$ is the $n^{\text {th }}$ derivative of $f(y)$ at point $y=T$ for $n>0$. To have a consistent notation, we define $f^{(0)}(T)=$ $f(T)$. This results in the following relationship between the error in computing $P_{e}$ and the error in estimating Cumulants:

$$
\Delta P_{e} \simeq \sum_{n=1}^{\infty} \Delta k_{n} \frac{i^{n-1}}{n !} f^{(n-1)}(0) .
$$

In order to simplify (27), we assume that the derivatives, $f^{(n)}(y)$, are similar to the derivatives of the normal density. This is based on the fact that pdf of the bit LLR is close to the normal density. Thus we suppose that

$$
f^{(n)}(y) \simeq(-1)^{n} e^{-y^{2} / 2} T_{n}(y),
$$

where $T_{n}(y)$ is the Hermite polynomial [14] of order $n$, defined as

$$
T_{n}(y)=\sum_{j=0}^{\lfloor n / 2\rfloor} \frac{(-1)^{j} n !}{2^{j}(n-2 j) ! j !} y^{n-2 j}
$$

This approximation results in the following equation:

$$
\Delta P_{e} \simeq \sum_{l=0}^{\infty} \frac{\Delta k_{2 l+1}}{(2 l+1) 2^{l} l !}
$$

Numerical values presented in Table-3, section $\mathrm{V}$ have been calculated using (30).

\section{Numerical Results}

The proposed algorithm is compared with Monte-Carlo (MC) simulation in this section. A Turbo-code of length 100 and rate $1 / 2$ is used to perform the simulations as seen in Figure 1. It is evident that increasing the number of Cumulants (the order of approximation) that are involved from two to four significantly improves the approximation. A short code is used to assure the accuracy of MC simulation and hence provide a reliable comparison benchmark. The relationship between interval Probabilities of the Point Estimates (PPE) and the number of samples $n$ is computed using numerical methods (complete description of these methods are available in the Journal version of this paper [24]) and demonstrated in Table1 and Table-2. It is evident that the proposed method is still accurate even by using fewer samples compared to the MC simulations.

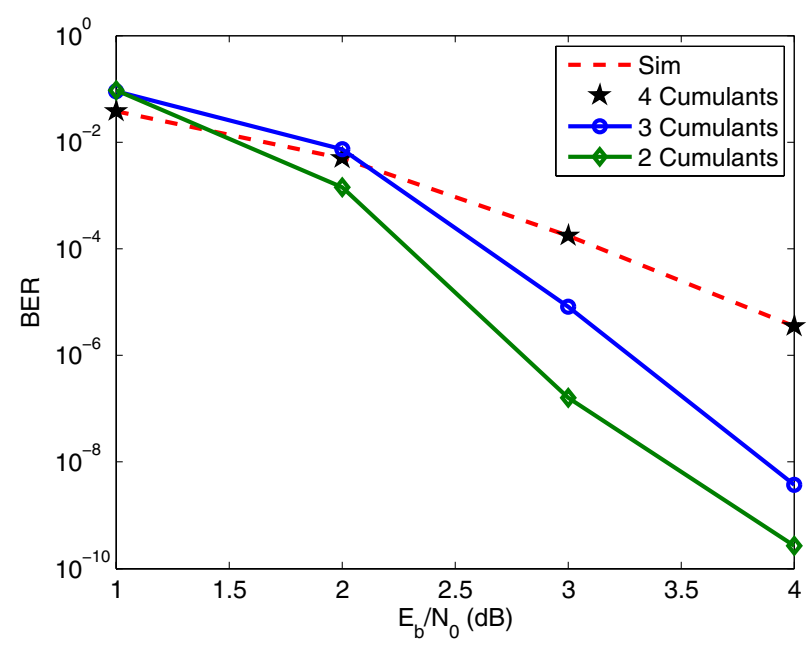

Fig. 1. BER curves for Turbo-Code of length 100 and rate $1 / 2$.

\begin{tabular}{|c||c|c|c|}
\hline$n$ & $\theta$ & $p_{c}$ & $p_{m}$ \\
\hline \hline $10^{4}$ & 0.0060 & 0.95 & 0.67 \\
\hline $10^{5}$ & 0.0060 & 0.96 & 0.70 \\
\hline $10^{6}$ & 0.0060 & 0.97 & 0.96 \\
\hline \hline $10^{4}$ & 0.0020 & 0.94 & 0.66 \\
\hline $10^{5}$ & 0.0020 & 0.95 & 0.70 \\
\hline $10^{6}$ & 0.0020 & 0.96 & 0.96 \\
\hline \hline $10^{4}$ & 0.0005 & 0.93 & 0.33 \\
\hline $10^{5}$ & 0.0005 & 0.94 & 0.68 \\
\hline $10^{6}$ & 0.0005 & 0.95 & 0.95 \\
\hline
\end{tabular}

Table 1 : The Relationship between $n$ (number of samples) and the PPEs $\left(p\left(\left|\Delta P_{e}\right|<\theta\right)\right)$ at $E_{b} / N_{0}=2 \mathrm{~dB}$ for the Cumulant method $p_{c}$ and the MC simulation $p_{m}$.

\begin{tabular}{|c||c|c|c|}
\hline$n$ & $\theta$ & $p_{c}$ & $p_{m}$ \\
\hline \hline $10^{6}$ & $10^{-6}$ & 0.98 & 0.27 \\
\hline $10^{7}$ & $10^{-6}$ & 0.99 & 0.96 \\
\hline $10^{8}$ & $10^{-6}$ & $\approx 1$ & $\approx 1$ \\
\hline \hline $10^{6}$ & $5 \times 10^{-7}$ & 0.94 & 0 \\
\hline $10^{7}$ & $5 \times 10^{-7}$ & 0.96 & 0.68 \\
\hline $10^{8}$ & $5 \times 10^{-7}$ & $\approx 1$ & 0.99 \\
\hline \hline $10^{6}$ & $10^{-7}$ & 0.91 & 0 \\
\hline $10^{7}$ & $10^{-7}$ & 0.92 & 0.08 \\
\hline $10^{8}$ & $10^{-7}$ & 0.94 & 0.49 \\
\hline
\end{tabular}

Table 2 : The Relationship between $n$ (number of samples) and the PPEs $\left(p\left(\left|\Delta P_{e}\right|<\theta\right)\right)$ at $E_{b} / N_{0}=4 \mathrm{~dB}$ for the Cumulant method $p_{c}$ and the MC simulation $p_{m}$.

This method is similar to the one introduced in [25], where a suitable model for the $p d f$ of bit $L L R$ is suggested. The moment matching method with maximum entropy principle is then used to estimate the parameters of the suggested model for the $p d f$. In this case, a constrained maximization problem is solved using iterative Newton-Raphson method. At each 
iteration, solving a system of linear equations (with the same degree as the number of moments) as well as evaluating an integral of the exponential form is required. This renders the Cumulant method proposed here significantly less complex as compared to the moment method of [25].

Table-3 provides an example on how to decide on the required accuracy in estimating the Cumulants of different orders. Numerical values presented have been calculated using (30).

\begin{tabular}{|c|c|c|c|c|}
\hline$l$ & 0 & 1 & 2 & $\Delta P_{e}$ \\
\hline $2 l+1$ & 1 & 3 & 5 & - \\
\hline$\Delta k_{2 l+1}$ & $10^{-4}$ & $10^{-3}$ & $10^{-2}$ & $5.17 \times 10^{-4}$ \\
\hline
\end{tabular}

Table 3 : The Relationship between error in Cumulant estimation and the error in BER estimation.

\section{CONCLUding Remarks}

The problem of performance evaluation of a coded communication system with bit decoding algorithms in low BER regions is considered. The main ingredient of a bit decoding algorithm is the reliability information, i.e. the $L L R$. The $p d f$ of the bit $L L R$ is estimated using Cumulant matching technique. This method is based on estimating the characteristic function of the bit $L L R$ using its Cumulants. In order to have an unbiased estimation of the Cumulants with minimum variance, the best choices are the $K$-statistics. Once the characteristic function of a random variable is known, the rest of the $p d f$ computation is straightforward using the IFT. Numerical results demonstrate a close agreement between the theory and simulations. It is also shown that the error in BER estimation is bounded and may be reduced by increasing the accuracy of Cumulant estimation or equivalently increasing the number of samples. The complexity of this method is similar to the Monte-Carlo simulation with the advantage of providing similar accuracy using significantly fewer samples. The time consuming part of performance analysis is sample generation which is significantly decreased using the proposed method. Processing the samples and estimating the BER can be done very fast. Specifically in the provided example, 1 million samples are processed in less than a minute using an ordinary PC with AMD-Athlon 64-bit $3 \mathrm{GHz}$ Processor running Windows XP.

\section{REFERENCES}

[1] K. Hinton, G. Nicholson,"Probability Density Function for the Phase and Frequency Noise in a Semiconductor Laser," IEEE Journal of Quantum Electronics, vol. 22, no. 11, pp. 2107-2115, November 1986.

[2] D. Hatzinakos, C. L. Nikias, "Estimation of Multi-path Channel Response in Frequency Selective Channels," IEEE Journal on selected areas in communications, vol. 7, no. 1, pp. 12-19, January 1989.

[3] L. X. Wang, J. M. Mendel, "Cumulant-Based Parameter Estimation Using Structured Networks," IEEE Transactions on Neural Networks, vol. 2, no. 1, pp. 73-83, January 1991.

[4] A. G. Stogioglou, S. McLaughlin, "MA Parameter Estimation and Cumulant Enhancement," IEEE Transactions on Signal Processing, vol. 44, no. 7, pp. 1704-1718, July 1996.

[5] A. K. Nandai, "Robust Estimation of Third-Order Cumulants in Applications of Higher-Order Statistics," IEE Proceedings-F, vol. 140, no. 6, pp. 380-389, December 1993.
[6] B. C. Y. Wong, I. F. Blake, "Detection in Multivariate Non-Gaussian Noise," IEEE Transactions on Communications, vol. 42, no. 2/3/4, pp. 1672-1683, February/March/April 1994.

[7] Y. Zhao, X. Zhuang, S. J. Ting, "Gaussian Mixture Modeling of NonGaussian Source for Autoregressive Process," IEEE Transactions on Signal Processing, vol. 43, no. 4, pp. 894-903, April 1995.

[8] J. K. Tugnait, "Blind Equalization and Estimation of Digital Communication FIR Channel Using Cumulant Matching," IEEE Transactions on Communications, vol. 43, no. 2/3/4, pp. 1240-1245, February/March/April 1995.

[9] J. K. Tugnait, U. Gummadavelli, "Blind Equalization and Channel Estimation with Partial Response Input Signals," IEEE Transactions on Communications, vol. 45, no. 9, pp. 1025-1031, September 1997.

[10] L. Chen, H. Kusaka, M. Kominami, "Blind Phase Recovery in QAM Communication System Using Higher Order Statistics," IEEE Signal Processing Letters, vol. 3, no. 5, pp. 147-149, May 1996.

[11] S. Chen, Y. Wu, and S. Mclaughlin, "Genetic Algorithm Optimization for Blind Channel Identification with Higher Order Cumulant Fitting," IEEE Transactions on Evolutionary Computation, vol. 1, no. 4, pp. 259265, November 1997.

[12] M. Martone, "Cumulant-Based Adaptive Multichannel Filtering for Wireless Communication Systems with Multi-path RF Propagation Using Antenna Arrays," IEEE Transactions on Vehicular Technology, vol. 47, no. 2, pp. 377-391, May 1998.

[13] A. Abel, W. Schwarz, M. Gotz, "Noise Performance of Chaotic Communication Systems," IEEE Transactions on Circuits and Systems, vol. 47, no. 12, pp. 1726-1732, December 2000.

[14] A. Abedi, A. K. Khandani, "An Analytical Method for Approximate Performance Evaluation of Binary Linear Block Codes, "IEEE Transactions on Communications, vol. 52, no. 2, pp. 228-235, February 2004.

[15] C. Berrou, A. Glavieux and P. Thitimajshima, "Near Shannon Limit Error-Correcting Coding and Decoding: Turbo-Codes (1)," Proceedings of IEEE International Conference on Communications, Geneva, Switzerland, pp. 1064-1070, May 1993.

[16] D. Divsalar, S. Dolinar and F. Pollara, "Iterative Turbo Decoder Analysis Based on Density Evolution," IEEE Journal on Selected Areas in Communications, vol. 19, no. 5, pp. 891-907, May 2001.

[17] H. El-Gamal and A. R. Hammons Jr., "Analyzing the Turbo Decoder Using the Gaussian Approximation," IEEE Transactions on Information Theory, vol. 47, no. 2, pp. 671-686, February 2001.

[18] A. Abedi and A. K. Khandani, "Invariance Properties of Binary Linear Block Codes over Memoryless Channels with Discrete Input," IEEE Transactions on Information Theory, vol. 53, no. 3, pp. 1215-1218, March 2007.

[19] M. Abramowitz, and I. A. Stegun, (Eds.). Handbook of Mathematical Functions with Formulas, Graphs, and Mathematical Tables, 9th printing. New York: Dover, p. 928, 1972.

[20] J. F. Kenney, and E. S. Keeping, Mathematics of Statistics, Pt. 2, 2nd ed. Princeton, NJ: Van Nostrand, 1951.

[21] P. R. Halmos, "The Theory of Unbiased Estimation." Ann. Math. Stat. 17, pp. 34-43, 1946.

[22] C. Rose, and M. D. Smith, "k-Statistics: Unbiased Estimators of Cumulants." C:7.2C in Mathematical Statistics with Mathematica. New York: Springer-Verlag, pp. 256-259, 2002.

[23] A. Stuart, J. K. Ord, Kendall's Advance Theory of Statistics, vol. 1, pp. 418-421, sixth Ed., John Wiley, 1994.

[24] A. Abedi, M. E. Thompson, A. K. Khandani, "Application of Cumulant Method In Performance Evaluation of Turbo-Like Codes," IEEE Transactions on Communications, Accepted, To appear in 2007.

[25] A. Abedi, A. K. Khandani, "A New Method for Performance Evaluation of Bit Decoding Algorithms Using Statistics of the Log Likelihood Ratio," 4th International Symposium on Turbo-codes, April 2006, Munich, Germany 